\title{
DOA Estimation Method of Weak Signal under the Compound Background of Strong Interference and Colored Noise
}

\author{
Bin Lin $\mathbb{D}^{1},{ }^{1,2}$ Guoping Hu ${ }^{D},{ }^{1}$ Hao Zhou, ${ }^{1}$ Guimei Zheng, ${ }^{1}$ and Yuwei Song ${ }^{1}$ \\ ${ }^{1}$ Air and Missile Defense College, Air Force Engineering University, Xi'an, Shaanxi 710051, China \\ ${ }^{2}$ Graduate College, Air Force Engineering University, Xi'an, Shaanxi 710051, China \\ Correspondence should be addressed to Guoping Hu; hgp6068@163.com
}

Received 3 December 2021; Accepted 8 January 2022; Published 4 February 2022

Academic Editor: Qi Liu

Copyright (C) 2022 Bin Lin et al. This is an open access article distributed under the Creative Commons Attribution License, which permits unrestricted use, distribution, and reproduction in any medium, provided the original work is properly cited.

\begin{abstract}
The traditional algorithm performing direction of arrival (DOA) estimation under the background of strong interference and colored noise has the problems of low estimation accuracy and small measurement targets. Based on the construction of a fourthorder cumulant (FOC) matrix to suppress colored noise, this paper adopts the extended noise subspace (ENS) algorithm and the fixed projection blocking (FPB) algorithm to estimate the DOA of weak targets. Firstly, a FOC matrix of the received signal vector is established to curb the noise component, and the eigenvalue decomposition is performed. Then, two approaches of weak signal DOA estimation are proposed. One approach is to merge the space where the strong interference steering vector lies into the noise subspace to construct an extended noise subspace, and then, the multisignal classification (MUSIC) algorithm is used to obtain the DOA estimation of the weak signal on the basis of the extended noise subspace. Another approach is to build the orthogonal projection matrix of the interference subspace as the interference blocking matrix, and the receiving array signal is preprocessed, and on the basis of it, the eigen decomposition is performed again to obtain the DOA information of the weak signal. Both algorithms make breakthroughs in the aperture limitation of the traditional algorithm, effectively expand the aperture, and promote the accuracy of estimation. The simulation tests the effectiveness of the proposed method.
\end{abstract}

\section{Introduction}

With the increasing intricacy of the electromagnetic environment, radar detection is interference by more and more electromagnetic, making it increasingly difficult for radar to detect targets [1,2]. Strong electromagnetic interference will cover up the target's echo signal or overload the signal processor and receiver $[3,4]$. When the power of the interference signal is greater than that of the echo signal, the received data is directly used in the estimation of the direction of arrival, and the false peak resulting from strong interference will be regarded as the peak of the source, which will give birth to the misinterpretation of the correct angle $[5,6]$. Firstly, at present, one method to address this problem is signal separation, mainly including the Relaxation (Relax) algorithm [7] and the CLEAN algorithm [8]. This type of algorithm separates the output part of the array of all signals into multiple data blocks and then determines and removes the data blocks containing the interference signals according to the characteristics of the interference signal so as to achieve target recognition. However, this type of algorithm is extremely complex. Secondly, the jamming jam method (JJM) was proposed by Fang et al. and its extended application $[9,10]$. The core concept is to use the angle information of the signal receiving matrix to establish an interference blocking matrix to eliminate the interference signal part of the received signal covariance matrix. However, the algorithm requires a precise prediction of the direction of interference. In addition, there is an Extended Noise Subspace algorithm [11]. The algorithm first constructs an extended noise subspace for strong interferences and noises, and then performs conventional DOA estimation. Dong et al. [12] deduced an interference blocking method based on modified projection. The interference direction is not a necessity in this approach, and it is of more practical use. 
However, the abovementioned strong interference rejection algorithms are characterized by low estimation accuracy and small measurement targets in the direction finding under the background of nonideal colored noise. According to related literature, the calculation of high-order cumulants is blind Gaussian which can achieve aperture expansion and provides a powerful tool in response to Gaussian colored noise. Literature [13, 14] accordingly proposed a variety of FOC-based algorithms, which have better angle estimation performance under Gaussian colored noise conditions. Nonetheless, there are still some problems in terms of angular resolution and complexity. Tufail and Ahmed [15] used the FOC and ESPRIT algorithm to propose DOA estimation based on the genetic algorithm (GA) and obtained the multiple invariant cumulant ESPRIT algorithm, which has a better angular resolution, but the problem of excessive complexity remains unsolved. Literature [16] used the real-valued sparse Bayesian learning method to transform the FOC matrix into a real-valued matrix and simplify the algorithm through unitary transformation. The abovementioned FOC algorithm has made certain progress in the field of array signal processing, but the research on the background of strong interference is scarce.

To tackle the above problems, this paper puts forward the fourth-order cumulant-expanded noise subspace (FOCENS) algorithm and the fourth-order cumulant-fixed projection blocking (FOC-FPB) algorithm. One is to use the FOC matrix to substitute for the traditional covariance matrix. After eigenvalue decomposition, the space of the strong interference steering vector is incorporated into the noise subspace, and thus, the extended noise subspace is constructed. And the conventional MUSIC algorithm is used to obtain the DOA estimation of the weak signal on the basis of the extended noise subspace. The other method is to construct the orthogonal projection matrix of the strong interference signal subspace as the interference blocking matrix, and the received array signal is preprocessed. And the eigenvalue decomposition is performed again to obtain the noise subspace. Eventually, the MUSIC algorithm is used to search for spectral peaks to obtain the azimuth angle of the weak signal. Compared with traditional algorithms, this algorithm enjoys higher estimation accuracy under a variety of conditions.

\section{Signal Model and FOC Vector Formulation}

2.1. Signal Model. Assuming that $K$ far-field narrowband signals and $J$ strong interference signals $S_{i}(t)(i=1,2, \ldots, J$, $\ldots, K+J)$ are incident on a uniform linear array, the incident angle is $\theta_{i}$, and if the first $J$ is a strong interference signal, their power satisfies $\sigma_{1}^{2}>\sigma_{2}^{2}>\cdots>\sigma_{J}^{2} \gg \sigma_{J+1}^{2}>\cdots>$ $\sigma_{K+J}^{2}$. The distance $d$ of the array elements is half of the signal wavelength. The array is composed of $M$ array elements, each of which is omnidirectional. The received signal of the $m$-th array element of the array at time $t$ can be expressed as

$$
x_{m}(t)=\sum_{i=1}^{K+J} s_{i}(t) \exp \left\{j(m-1) \pi \sin \left(\theta_{i}\right)\right\}+n_{m}(t),
$$

where $n_{m}(t)$ is the Gaussian colored noise of the $m$-th element at time $t$ and $s_{i}(t)$ is the complex envelope of the $i$-th source.

Assuming that the plane wave propagates along a straight line, the array response vector corresponding to the direction can be expressed as

$\mathbf{a}\left(\theta_{i}\right)=\left[1, \exp \left(-j \pi \sin \theta_{i}\right), \ldots, \exp \left(-j \pi(M-1) \sin \theta_{i}\right)\right]^{\mathrm{T}}$,

among which, $(\cdot)^{\mathrm{T}}$ represents the transpose of the matrix.

The direction matrix is defined as

$$
\mathbf{A}(\theta)=\left[\mathbf{a}\left(\theta_{1}\right), \mathbf{a}\left(\theta_{2}\right), \ldots, \mathbf{a}\left(\theta_{J}\right), \ldots, \mathbf{a}\left(\theta_{\mathrm{K}+\mathrm{J}}\right)\right] .
$$

This matrix is a $M \times(K+J)$ dimensional direction matrix.

The vector output of the array element is expressed as

$$
\mathbf{X}(t)=\mathbf{A}(\theta) \mathbf{S}(t)+\mathbf{N}(t)
$$

In the above formula, $\mathbf{S}(t)$ is the $(K+J) \times 1$ dimensional signal vector and $\mathbf{N}(t)$ is the $\mathrm{M} \times 1$ dimensional Gaussian colored noise vector.

2.2. Observation Model Based on FOC Vector. Compared with the second-order cumulant, the high-order cumulant can better characterize the signal characteristics and has the blind Gaussian property. Therefore, in the actual array signal processing, the fourth-order cumulant is frequently used for processing.

Regarding a uniform linear array, according to the symmetric definition of the FOC of the zero-mean stable random process, the FOC of the array received data is

$$
\begin{aligned}
& \operatorname{cum}\left(x_{k_{1}}, x_{k_{2}}, x_{k_{3}}^{*}, x_{k_{4}}^{*}\right) \\
& =E\left(x_{k_{1}} x_{k_{2}} x_{k_{3}}^{*} x_{k_{4}}^{*}\right)-E\left(x_{k_{1}} x_{k_{3}}^{*}\right) E\left(x_{k_{2}} x_{k_{4}}^{*}\right) \\
& \quad-E\left(x_{k_{1}} x_{k_{4}}^{*}\right) E\left(x_{k_{2}} x_{k_{3}}^{*}\right)-E\left(x_{k_{1}} x_{k_{2}}\right) E\left(x_{k_{3}}^{*} x_{k_{4}}^{*}\right) .
\end{aligned}
$$

In the above formula, $(\cdot)^{*}$ represents conjugate and cum() represents cumulant.

According to literature [13], it is easy to get

$$
\begin{aligned}
\mathbf{C}_{x}= & E\left\{\left(\mathbf{X} \otimes \mathbf{X}^{*}\right)\left(\mathbf{X} \otimes \mathbf{X}^{*}\right)^{\mathrm{H}}\right\}- \\
& E\left\{\left(\mathbf{X} \otimes \mathbf{X}^{*}\right)\right\} E\left\{\left(\mathbf{X} \otimes \mathbf{X}^{*}\right)^{\mathrm{H}}\right\}-. \\
& E\left\{(\mathbf{X X})^{\mathrm{H}}\right\} \otimes E\left\{\left(\mathbf{X} \mathbf{X}^{\mathrm{H}}\right)^{*}\right\}
\end{aligned}
$$

In the above formula, $\otimes$ represents the Kronecker product.

Substituting the signal vector into the above formula can obtain the FOC matrix of the signal as follows:

$$
\begin{aligned}
\mathbf{C}_{\mathrm{s}}= & E\left\{\left(\mathbf{S} \otimes \mathbf{S}^{*}\right)\left(\mathbf{S} \otimes \mathbf{S}^{*}\right)^{\mathrm{H}}\right\} \\
& -E\left\{\left(\mathbf{S} \otimes \mathbf{S}^{*}\right)\right\} E\left\{\left(\mathbf{S} \otimes \mathbf{S}^{*}\right)^{\mathrm{H}}\right\} \\
& -E\left\{(\mathbf{S S})^{\mathrm{H}}\right\} \otimes E\left\{\left(\mathbf{S} \mathbf{S}^{\mathrm{H}}\right)^{*}\right\},
\end{aligned}
$$


where the signal FOC matrix $\mathbf{C}_{\mathrm{s}}$ is a $\mathrm{K}^{2} \times K^{2}$ complex matrix, and its $(i+1) K+j$ row and $(k-1) K+l$ column elements can be expressed as

$$
\operatorname{cum}\left(s_{i}, s_{j}, s_{k}^{*}, s_{l}^{*}\right), \forall i, j, k, l \in\{1,2, \ldots, \mathrm{K}\} .
$$

With respect to an independent signal source, according to the nature of the higher-order cumulant, the elements in the FOC $\mathrm{C}_{\mathrm{s}}$ of the signal are not zero if and only if $i=j=k=l$, and all other elements are zero, namely,

$$
\mathbf{C}_{\mathrm{s}}=\operatorname{cum}\left(s_{i}, s_{j}, s_{k}^{*}, s_{l}^{*}\right)\left\{\begin{array}{l}
\neq 0, i=j=k=l, \\
=0, \text { otherwise. }
\end{array}\right.
$$

Hence, there are only $K$ nonzero elements in $\mathbf{C}_{\mathrm{s}}$, and they are situated at position $(k-1) K+k(k=1,2, \ldots, K)$ on the diagonal of $\mathbf{C}_{s}$. Now, the rows and columns are deleted with all zero elements in $\mathbf{C}_{s}$; $\mathbf{C}_{\mathrm{s}}$ is reduced from the $\mathrm{K}^{2} \times K^{2}$ dimensional matrix to the $\mathrm{K} \times K$ dimensional diagonal matrix. Then, the simplified signal FOC matrix can be expressed as

$$
\mathbf{C}_{\mathrm{s}}=\operatorname{diag}\left(\gamma_{1}, \gamma_{2}, \ldots, \gamma_{k}\right) \text {. }
$$

In the above formula, $\gamma_{k}=\operatorname{cum}\left(s_{k}, s_{k}, s_{k}^{*}, s_{k}^{*}\right)$.

It can also be obtained that the FOC matrix of noise is

$$
\begin{aligned}
\mathbf{C}_{n}= & E\left\{\left(\mathbf{N} \otimes \mathbf{N}^{*}\right)\left(\mathbf{N} \otimes \mathbf{N}^{*}\right)^{H}\right\} \\
& -E\left\{\mathbf{N} \otimes \mathbf{N}^{*}\right\} \cdot E\left\{\left(\mathbf{N} \otimes \mathbf{N}^{*}\right)^{H}\right\} \\
& -E\left\{\mathbf{N} \cdot \mathbf{N}^{H}\right\} \otimes E\left\{\left(\mathbf{N} \cdot \mathbf{N}^{H}\right)^{*}\right\} .
\end{aligned}
$$

In addition, the array steering vector after array expansion according to the FOC defined above is

$$
\mathbf{b}(\theta)=\mathbf{a}(\theta) \otimes \mathbf{a}^{*}(\theta) .
$$

The expanded direction matrix is

$$
\begin{aligned}
\mathbf{B}(\theta) & =\left[\begin{array}{llll}
\mathbf{b}\left(\theta_{1}\right) & \mathbf{b}\left(\theta_{2}\right) & \cdots & \mathbf{b}\left(\theta_{k}\right)
\end{array}\right] \\
& =\left[\begin{array}{ll}
\mathbf{a}\left(\theta_{1}\right) \otimes \mathbf{a}^{*}\left(\theta_{1}\right) \mathbf{a}\left(\theta_{2}\right) \otimes \mathbf{a}^{*}\left(\theta_{2}\right) \cdots \mathbf{a}\left(\theta_{K}\right) \otimes \mathbf{a}^{*}\left(\theta_{K}\right)
\end{array}\right] .
\end{aligned}
$$

If each signal source is completely independent, the following formula holds [17]:

$$
\mathbf{C x}=\mathbf{B}(\theta) \mathbf{C}_{\mathrm{s}} \mathbf{B}^{\mathrm{H}}(\theta)+\mathbf{C} n .
$$

Assuming that the noise is Gaussian noise, whether it is white noise or colored noise with unknown spectral characteristics, the theoretical definition by the above formula $\mathbf{C}_{n}$ should always be a zero matrix. Due to noise deviation from Gaussian or finite data length calculation accuracy, $\mathbf{C}_{n}$ is a matrix approaching $\boldsymbol{0}$.

\section{DOA Estimation Based on FOC Vector}

Eigenvalue decomposition is performed on $\mathbf{C} x$, and its eigenvalues are arranged from large to small as $\lambda 1, \lambda 2, \ldots, \lambda_{M^{2}}$, and the corresponding eigenvector is $\mathbf{e}_{1}, \mathbf{e}_{2}, \ldots, \mathbf{e}_{M^{2}}$, where the eigenvectors corresponding to the $J$ large eigenvalues of the matrix $\mathbf{C} x$ are transformed into a fourth-order strong interference signal subspace [18-20]:

$$
\mathbf{E}_{J}=\left[\mathbf{e}_{1}, \mathbf{e}_{2}, \ldots, \mathbf{e}_{J}\right] .
$$

The eigenvectors corresponding to the $K$ larger eigenvalues are transformed into a fourth-order signal subspace:

$$
\mathbf{E}_{s}=\left[\mathbf{e}_{\mathrm{J}+1}, \mathbf{e}_{\mathrm{J}+2}, \ldots, \mathbf{e}_{\mathrm{J}+K}\right] .
$$

The eigenvectors corresponding to the other $M^{2}-K-J$ small eigenvalues are transformed into a fourth-order noise subspace:

$$
\mathbf{E}_{N}=\left[\mathbf{e}_{J+K+1}, \mathbf{e}_{J+K+2}, \ldots, \mathbf{e}_{M^{2}}\right]
$$

3.1. Expanded Noise Subspace DOA Estimation. Taking the MUISC algorithm as an example, the spatial spectrum value of a certain angle is the reciprocal of the projection modulus of the steering vector in direction to the projection space, that is, the noise subspace [21-23]. The peak value represents that the steering vector at this angle projected in the noise subspace is smaller than its surrounding angle. The direction steering vector is merged into the noise subspace to form an expanded noise subspace [24]. When the expanded noise subspace is used for spectrum estimation, there must be no peak in this direction, and the steering vector of the weak signal will be in the expanded noise subspace. The projection of the steering vector of the weak signal on the extended noise subspace is a small nonzero value, and its reciprocal will generate a larger peak so that strong interference can be curbed, and the DOA of a weak signal can be estimated [11].

Accordingly, the feature vector of the interference signal can be incorporated into the noise subspace to form an extended noise subspace, namely,

$$
\mathbf{E}_{J N}=\left[\mathbf{e}_{1}, \mathbf{e}_{2}, \ldots, \mathbf{e}_{J}, \mathbf{e}_{J+\mathrm{K}+1}, \ldots, \mathbf{e}_{M^{2}}\right] .
$$

Based on the interference-noise subspace, since the weak signal steering vector and the interference signal steering vector are often not orthogonal, in order to ensure the correct formation of the weak signal peak, the steering vector is transformed as follows:

$$
\mathbf{c}(\theta)=\left(\mathbf{I}-\mathbf{E}_{J} \mathbf{E}_{J}^{\mathrm{H}}\right) \mathbf{b}(\theta),
$$

where $\mathbf{b}(\theta)$ is the steering vector after the expansion of the FOC.

The conventional MUSIC method is adopted to search for spectral peaks and estimate the DOA of the weak signal:

$$
P_{1}(\theta)=\frac{\mathbf{c}^{\mathrm{H}}(\theta) \mathbf{c}(\theta)}{\mathbf{c}^{\mathrm{H}}(\theta) \mathbf{E}_{J N} \mathbf{E}_{J N}^{\mathrm{H}} \mathbf{c}(\theta)} .
$$

In the above formula, since the total signal subspace is orthogonal to the noise subspace, there is $\mathbf{E}_{J}^{\mathrm{H}} \mathbf{E}_{N}=0$. If $\theta_{i}$ is the incident angle of the weak signal, then

$$
\mathbf{E}_{N}\left(\mathbf{I}-\mathbf{E}_{J} \mathbf{E}_{J}^{\mathrm{H}}\right) \mathbf{a}\left(\theta_{i}\right)=\mathbf{E}_{N} \mathbf{a}\left(\theta_{i}\right)-0=0 .
$$


So the weak signal steering vector is orthogonal to the interference-noise subspace after transformation by equation (20).

Finally, the angle corresponding to the maximum point obtained through the spatial spectrum is the incident direction of the weak signal.

According to the above analysis, the FOC-ENS algorithm is summarized, as shown in Table 1.

\subsection{Modified Projection Blocking Method DOA Estimation.} After the division of the strong interference signal subspace, signal subspace, and noise subspace according (after formulas (15)-(17)) to the subspace theory, a modified projection blocking matrix $\boldsymbol{G}$ orthogonal to the strong interference signal subspace is established [12] as follows:

$$
\mathbf{G}=\mathbf{I}-\mathbf{E}_{J}\left(\mathbf{E}_{J}^{\mathrm{H}} \mathbf{E}_{J}\right)^{-1} \mathbf{E}_{J} \text {. }
$$

The global subspace is performed to get

$\mathbf{D}=\mathbf{G E}=\mathbf{G}\left[\mathbf{E}_{J}, \mathbf{E}_{S}, \mathbf{E}_{N}\right]=\left[\mathbf{0}_{J}, \mathbf{D}_{K+1}, \mathbf{D}_{K+2}, \ldots \mathbf{D}_{M^{2}}\right]$.

That is, the matrix $\boldsymbol{D}$ after the modified projection blocking transformation is merely related to the weak signal and noise and has nothing to do with the interference, achieving the suppression of the strong interference. Therefore, in order to avoid interference, the transformed data vector should be

$$
\mathbf{Y}=\mathbf{G C} x .
$$

The data covariance matrix after modified projection transformation is

$$
\mathbf{R}_{\mathrm{Y}}=\mathbf{G E R}_{\mathrm{s}} \mathbf{E}^{\mathrm{H}} \mathbf{G}^{\mathrm{H}}+\sigma^{2} \mathbf{G G}^{\mathrm{H}} .
$$

After the modified projection transformation, the strong interference is blocked, thereby eliminating the influence of the strong interference on the weak signal. Then, eigenvalue decomposition is performed on the receiving matrix again to obtain a new signal subspace $\mathbf{E}_{S}^{\prime}$ and noise subspace $\mathbf{E}_{N}^{\prime}$. At this time, $\mathbf{E}_{S}^{\prime}$ is only correlated with the weak signal, $\mathbf{E}_{N}^{\prime}$ orthogonal to the steering vector in the direction of the weak signal, and the spatial spectrum function is used as follows [25-27]:

$$
P_{2}(\theta)=\frac{1}{\mathbf{b}^{\mathrm{H}}(\theta) \mathbf{E}_{N}^{\prime} \mathbf{E}_{N}^{\prime} \mathrm{Hb}(\theta)} .
$$

Finally, the azimuth angle of the weak signal can be estimated with the help of the spectral peak search.

According to the above analysis, the algorithm of this paper is summarized, as depicted in Table 2.

\section{Simulation Results and Analysis}

Suppose the signal-to-noise ratio of the signal is defined as ${ }_{10 \log _{10}}\left(\sigma_{k}^{2} / \sigma_{n}^{2}\right)$, the interference-to-signal ratio is defined as $10 \log _{10}\left(\sigma_{\mathrm{j}}^{2} / \sigma_{\mathrm{k}}^{2}\right)$, and the interference-to-noise ratio is defined as $10 \log _{10}\left(\sigma_{\mathrm{j}}^{2} / \sigma_{\mathrm{n}}^{2}\right)$. Among them, $\sigma_{\mathrm{k}}^{2}$ is the power of the $k$-th signal, $\sigma_{j}^{2}$ is the power of the strong interference signal, and $\sigma_{\mathrm{n}}^{2}$ is the noise power.
The root mean square error (RMSE) is

$$
R M S E=\sqrt{\frac{1}{K N} \sum_{i=1}^{N} \sum_{k=1}^{K}\left(\left|\theta_{i}-\theta_{i k}\right|^{2}\right)} .
$$

Among them, $N$ is the number of Monte-Carlo experiments, $K$ is the number of weak signals, and $\theta_{i}$ and $\theta_{i k}$ are the true value and estimated value of the azimuth angle of the $i$-th signal in the $k$-th experiment.

4.1. Spatial Spectrum Estimation. Experiment 1 sets the incident angles of 3 target signals at $-30^{\circ}, 0^{\circ}$, and $30^{\circ}$, respectively; the incident angles of 2 strong interference signals are $-60^{\circ}$ and $60^{\circ}$; the number of array elements is 10; the signal-to-noise ratio (SNR) is $10 \mathrm{~dB}$; the signal-to-interference ratio (SIR) is $30 \mathrm{~dB}$; the number of snapshots is 200 . The advantages of the proposed algorithm in DOA estimation accuracy are analyzed. Figure 1 illuminates the spatial spectrum curves of the conventional FOC-MUSIC algorithm, JJM algorithm, FOC-ENS algorithm, and FOC-FPB algorithm.

Figure 1 illustrates that the conventional FOC-MUSIC algorithm is inclined to regard the false peak formed by the strong interference signal as the peak of the real target, thus failing to estimate the arrival angle of the target. Although the JJM algorithm can suppress strong interference signals, it has a mediocre effect on the estimation of the target's arrival angle, and it requires precise prediction of the direction of the strong interference signal. The FOC-ENS algorithm can better estimate the direction of arrival of the target. However, the direction of strong interference will exist during the estimation of small spikes, which will affect DOA estimation under certain conditions. The FOC-FPB algorithm can determine the target wave arrival angle best.

4.2. Comparative Analysis of Errors of Different Algorithms. Experiment 2 sets the number of array elements at 10, the SIR is $30 \mathrm{~dB}$, and the number of Monte Carlo experiments is 500. The errors of different algorithms in the Monte Carlo simulation experiment are also analyzed. Figure 2(a) illuminates that under the condition of $S N R=10 \mathrm{~dB}$, the number of snapshots varies from 50 to 500 in step of 50 and the variation curves of the RMSE of the JJM algorithm, ENS algorithm, FPB algorithm, FOC-ENS algorithm, and FOCFPB algorithm with the number of snapshots. Figure 2(b) shows the curve of the RMSE of the five algorithms changing from $-10 \mathrm{~dB}$ to $10 \mathrm{~dB}$ in step of $2 \mathrm{~dB}$ under the condition of 200 snapshots.

It can be inferred from Figure 2 that the RMSE of these methods dwindles as the SNR and the number of snapshots grow. In a composite background, when the number of snapshots and the SNR climb to a certain extent, the RMSE of several algorithms tends to be stable. Due to the suppression of spatial colored noise, the FOC-ENS algorithm and the FOC-FPB algorithm are better than other algorithms in low SNR. 
TABLE 1: FOC-ENS algorithm basic steps.

FOC-ENS algorithm

Step 1: estimate the data covariance matrix R of the array from the output vector $\mathbf{X}(t)$ of the array element

Step 2: perform eigen decomposition on the covariance matrix $\mathbf{C} x$ and arrange the eigenvalues in descending order so as to obtain interference subspace $\mathbf{E}_{J}$, signal subspace $\mathbf{E}_{s}$, and noise subspace $\mathbf{E}_{N}$

Step 3: take a set of orthogonal bases $\mathbf{E}_{J}$ determined by interference in the total signal subspace and merge it into the noise subspace $\mathbf{E}_{N}$ to form the interference-noise subspace $\mathbf{E}_{J N}$

Step 4: based on the interference-noise subspace $\mathbf{E}_{J N}$, perform a spectral peak search on $P_{1}(\theta)$ to estimate the DOA of the weak signal

TABLE 2: FOC-FPB algorithm basic steps.

FOC-FPB algorithm

Step 1 and Step 2 are the same as the FOC-ENS algorithm

Step 3: construct a modified projection matrix $\mathbf{G}$ orthogonal to the interference steering vector

Step 4: preprocess the received data vector to get $\boldsymbol{Y}$

Step 5: similarly duplicate Step 2 to do the second eigenvalue decomposition to get the noise subspace $\mathbf{E}_{N}^{\prime}$

Step 6: perform a spectral peak search on $P_{2}(\theta)$ and estimate the DOA of the weak signal

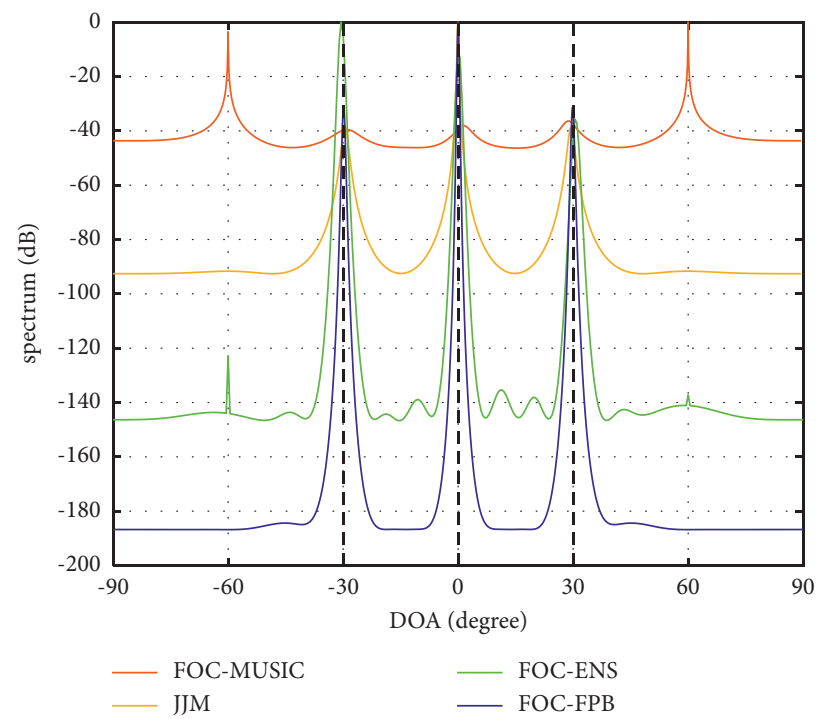

FIgURE 1: Comparison of spatial spectrum curves.

4.3. Comparative Analysis of Errors under Different Numbers of Interference Sources. Experiment 3 sets the number of array elements at 10 , the SIR is $30 \mathrm{~dB}$, the number of snapshots is 200, and the number of Monte Carlo experiments is 500. The RMSE of the proposed algorithm under different numbers of interference sources is analyzed. Figure 3(a) shows the SNR of the FOC-ENS algorithm from $-10 \mathrm{~dB}$ to $10 \mathrm{~dB}$ in step of $2 \mathrm{~dB}$ and the curves of the RMSE with the SNR under the different numbers of interference sources. Figure 3(b) depicts the FOC-FPB algorithm SNR changes from $-10 \mathrm{~dB}$ to $10 \mathrm{~dB}$ in step of $2 \mathrm{~dB}$, reflecting the variation curves of the RMSE with the SNR under the different numbers of interference sources.

As presented in Figure 3, the two algorithms can suppress interference and accurately estimate weak signals in a composite background. It effectively addresses the defect that the traditional algorithm cannot accurately estimate the weak signal under the background of strong interference and colored noise. As the number of interference sources increases, the direction finding performance of the proposed algorithm is slightly worse. The reason for the poor performance may be that the increase in the number of interferences gives rise to the leakage of the interference subspace, which affects the noise subspace.

4.4. Comparative Analysis of Errors under Different Noise Backgrounds. Experiment 4 sets the number of array elements at 10 , the SIR is $30 \mathrm{~dB}$, the number of snapshots is 200 , and the number of Monte Carlo experiments is 500. The RMSE under different noise backgrounds is analyzed. Figure 4(a) shows the SNR of the FOC-ENS algorithm from $-10 \mathrm{~dB}$ to $10 \mathrm{~dB}$ in step of $2 \mathrm{~dB}$ and the curves of the RMSE versus SNR under the two noise backgrounds. Figure 4(b) illuminates the FOC-FPB algorithm SNR changes from $-10 \mathrm{~dB}$ to $10 \mathrm{~dB}$ in step of $2 \mathrm{~dB}$, providing the curves of the RMSE with the SNR under different backgrounds.

Due to the unique blind Gaussian type of the FOC, the performance of this method under colored noise is similar to that under white noise. 


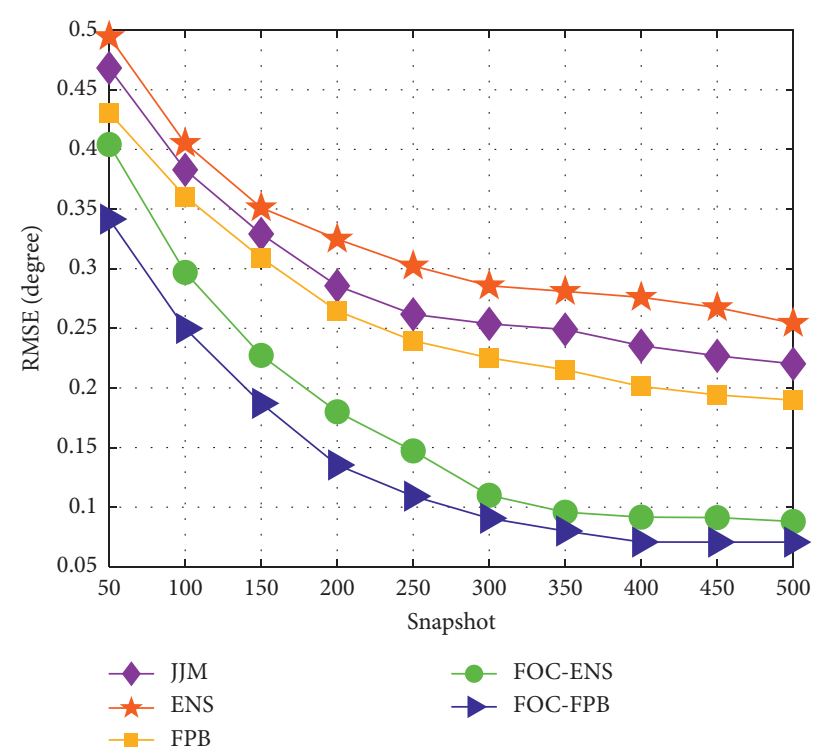

(a)

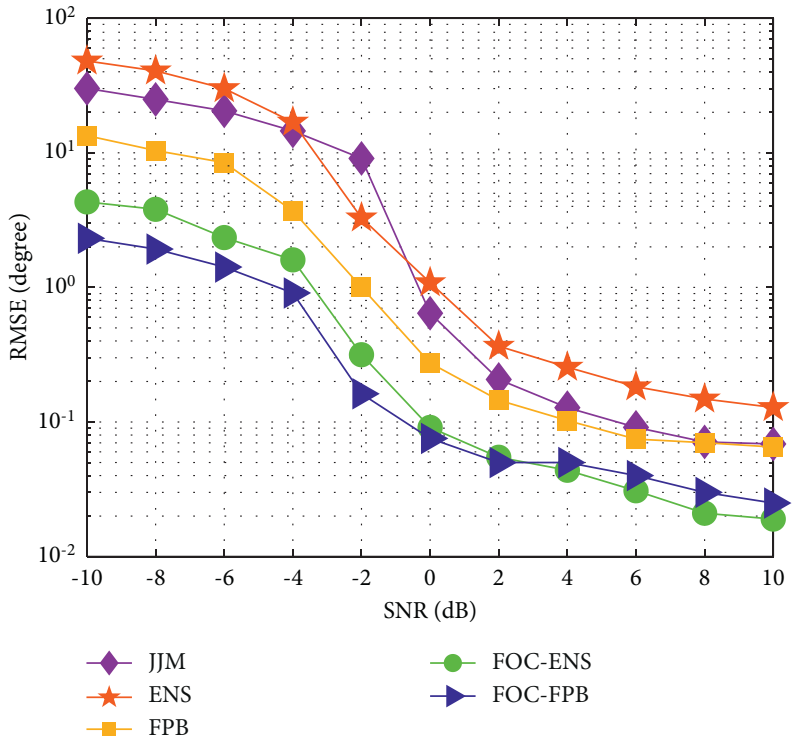

(b)

FIGURE 2: RMSE error analyses of different algorithms. (a) Relationship between RMSE and the number of snapshots. (b) Relationship between RMSE and SNR.

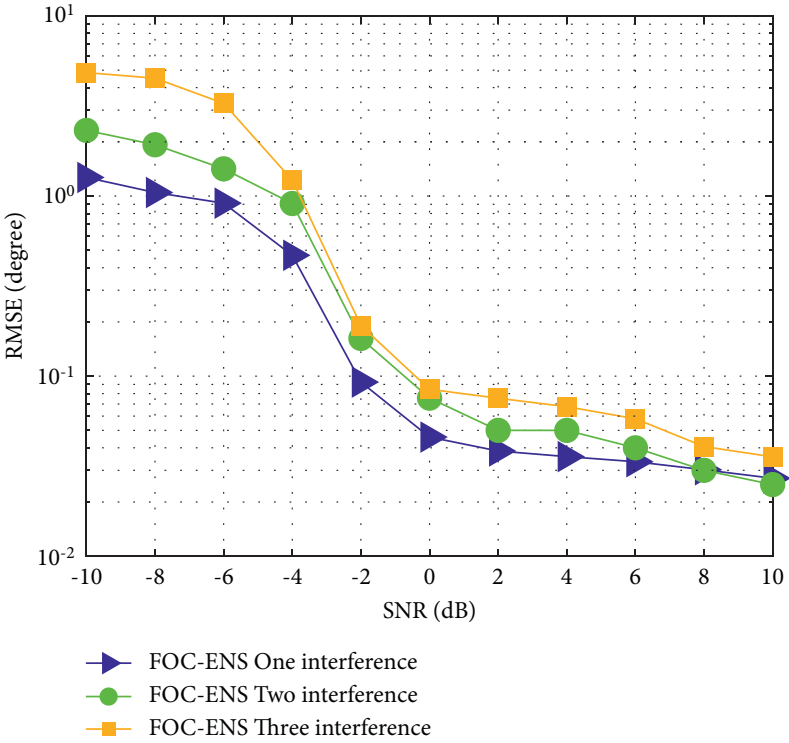

(a)

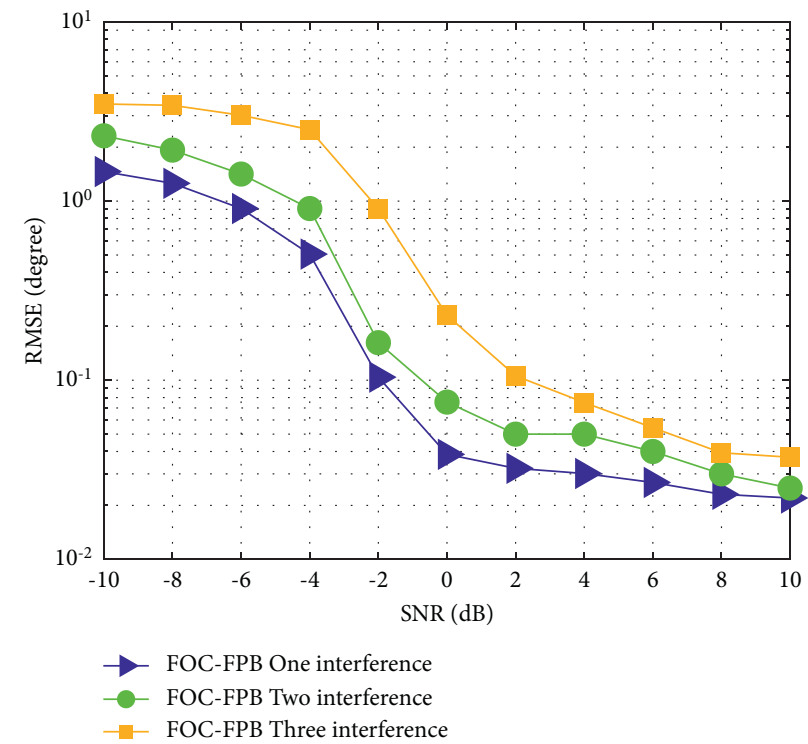

(b)

FIGURE 3: The statistical performance of the algorithm varying with SNR under different numbers of interference sources. (a) FOC-ENS algorithm. (b) FOC-FPB algorithm.

4.5. Algorithm Aperture Expansion Effect. Experiment 5 analyses the multitarget direction finding performance of the proposed algorithm. Figure 5(a) shows the simulation of the FOC-ENS algorithm. It sets the incidence angle of 12 target signals to be uniformly distributed from $-50^{\circ}$ to $60^{\circ}$, and the incidence angle of 2 strong interference signals to be $70^{\circ}$ and $80^{\circ}$, and other settings are the same as in Section 4.1. Figure 5(b) is the FOC-FPB simulation. It sets the incident angle of 11 target signals to be uniformly distributed from $-40^{\circ}$ to $60^{\circ}$, and the incident angle of 2 strong interference signals to be $-60^{\circ}$ and $-50^{\circ}$.

Owing to the aperture expansion characteristics of the FOC algorithm, the combined ENS algorithm and the FPB algorithm do not experience aperture loss, and the two improved algorithms perform well in multiobjective situations. 


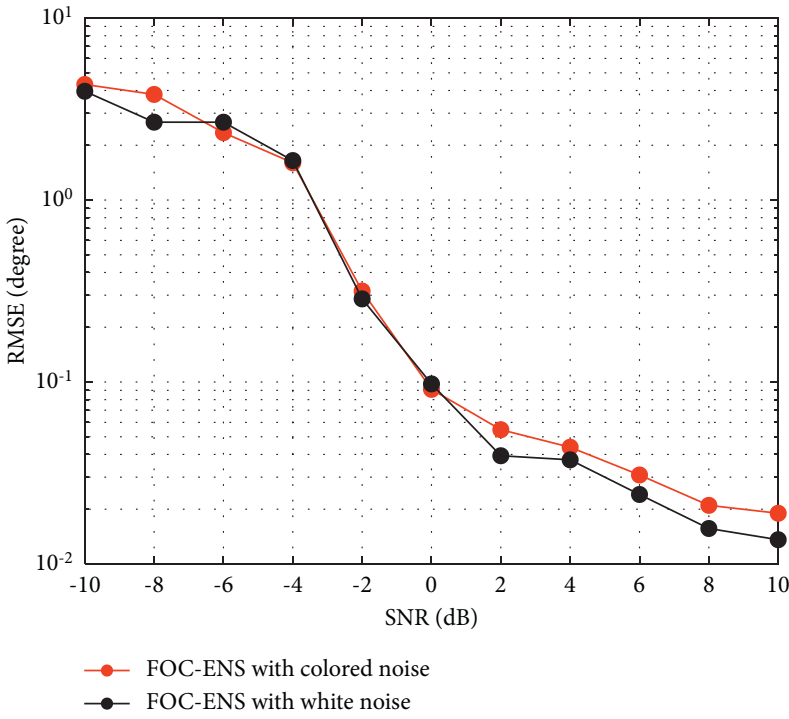

(a)

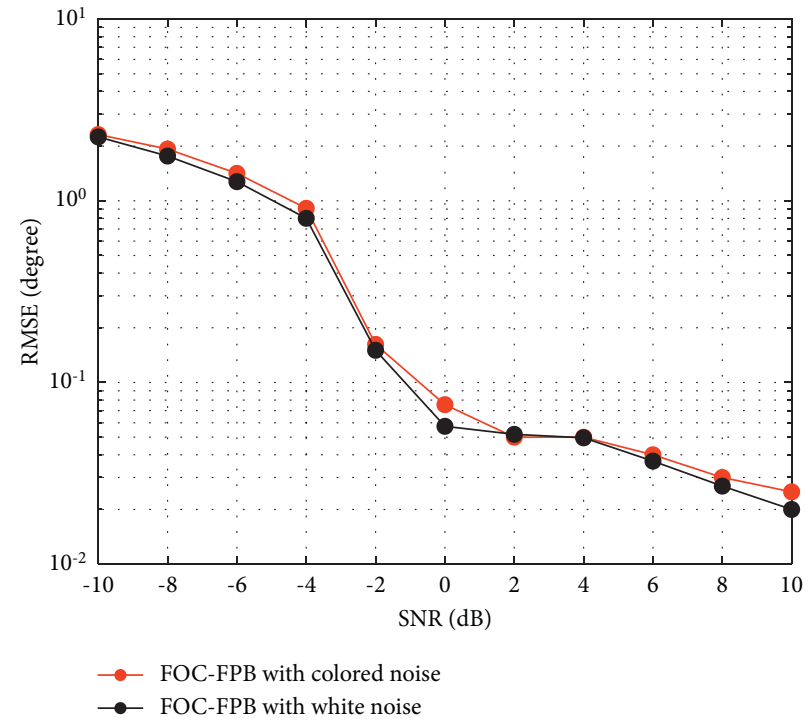

(b)

FIGURE 4: The statistical performance of different noise background algorithms varying with SNR. (a) FOC-ENS algorithm. (b) FOC-FPB algorithm.

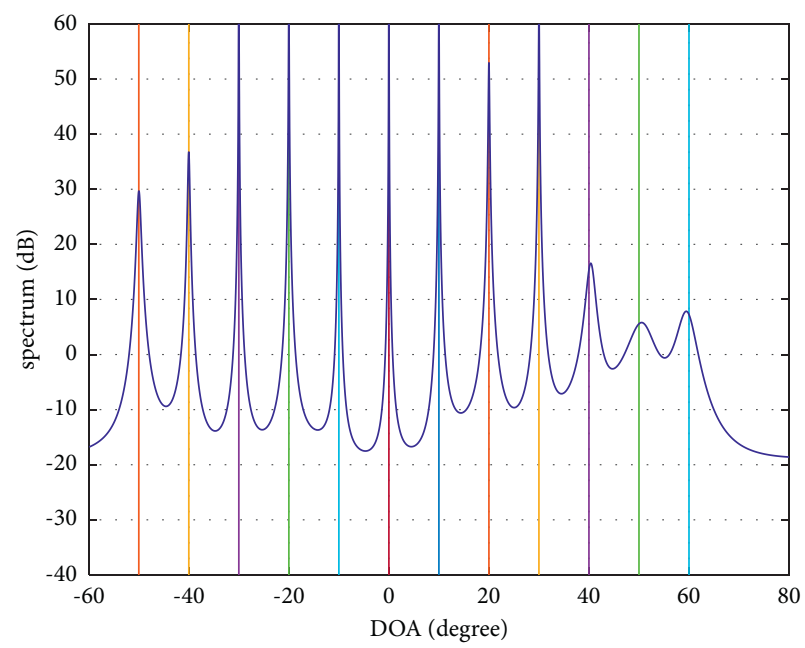

(a)

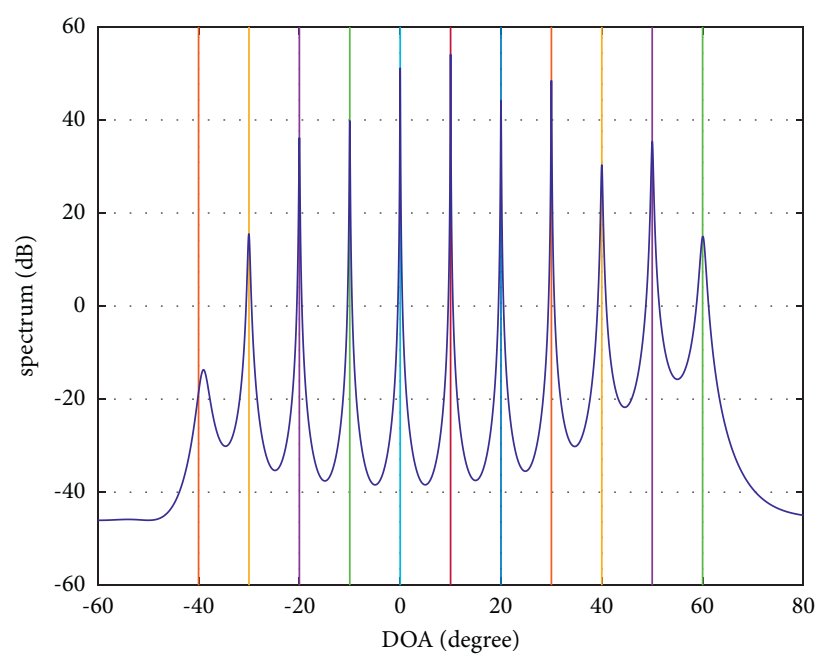

(b)

FIgUre 5: Multitarget direction [1] finding effect. (a) FOC-ENS algorithm. (b) FOC-FPB algorithm.

4.6. Time Complexity Analysis. Supposing that the number of sampling points is $N$, and the number of angle searches is $N_{\theta}$. The calculation amount of FOC-ENS and FOC-FPB algorithms mentioned in this paper is mainly the construction of the cumulant matrix, eigenvalue decomposition, and spectral peak search. The calculation amount is $O\left(9 N M^{4}+M^{6}+N_{\theta} M^{4}\right)$ and $O\left(9 N M^{4}+2 M^{6}+N_{\theta} M^{4}\right)$, respectively. Compared with the JJM and ENS algorithms with complexity $O\left(N M^{4}+M^{6}+N_{\theta} M^{4}\right)$ and the FPB algorithm with complexity $O\left(\mathrm{~N}^{4}+2 M^{6}+N_{\theta} M^{4}\right)$, the complexity is slightly higher.

Firstly, the calculation time of the two improved algorithms is simulated, and the number of target signals and the number of interference sources are set. $K$ represents the number of target, and $J$ represents the number of interference sources. Figures 6(a) and 6(b) show the simulation time comparison of FOC-ENS and FOC-FPB algorithms at different target numbers and interference sources, respectively. Then, $K=8$ and $J=2$ are set, and they are compared with traditional algorithms. Figure 6(c) shows the simulation time comparison of different algorithms.

It can be seen from Figure 6 that the FOC-ENS and FOCFPB algorithms construct FOC matrix, so the calculation time is slightly longer than that of the JJM, ENS, and FPB algorithms. FOC-ENS algorithm simulation time is shorter than the FOC-FPB algorithm in that the FOC-FPB algorithm has one more feature decomposition than the FOC-ENS algorithm, which enhances the complexity to a certain extent. 


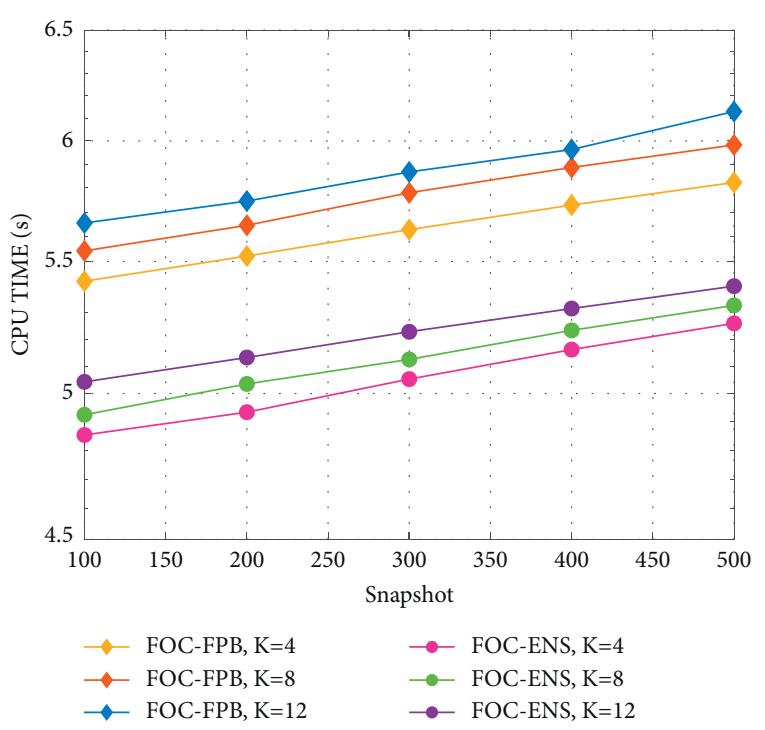

(a)

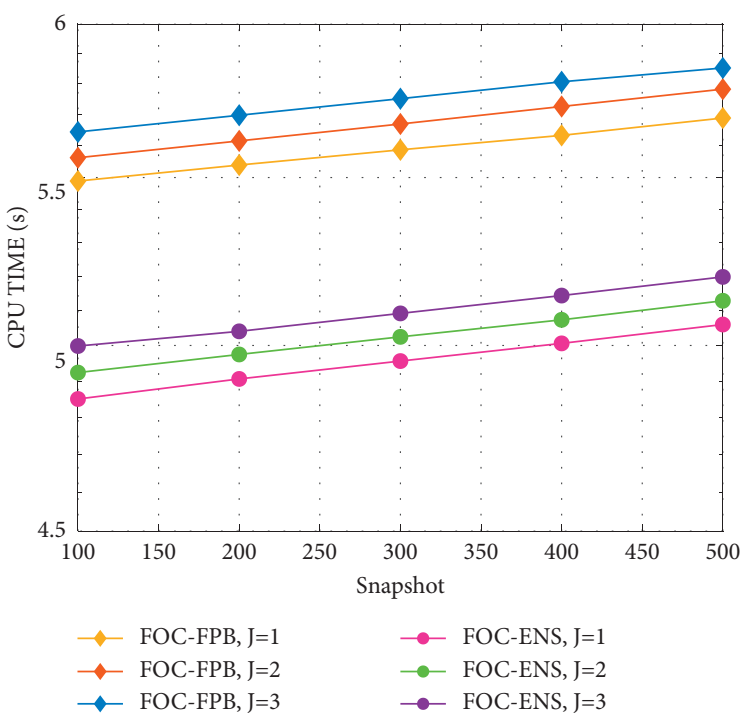

(b)

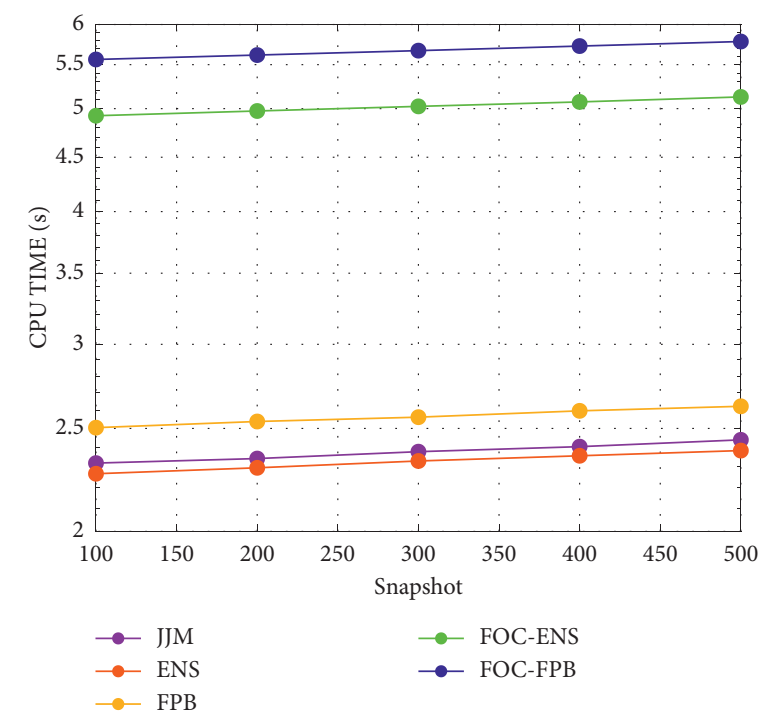

(c)

Figure 6: Comparison of simulation time. (a) Number of different targets. (b) Number of different interference sources. (c) Different algorithms.

\section{Conclusion}

To address the direction finding problem under the compound background of strong interference and colored noise, this paper proposes two algorithms based on FOC. Both algorithms need to construct a FOC matrix by receiving signal vectors to suppress noise components and then perform eigenvalue decomposition on the matrix and divide the subspace in accordance with the eigenvalues. The FOCENS algorithm merges the space where the strong interference steering vector is located into the noise subspace to construct an extended noise subspace for spectral peak search. The FOC-FPB algorithm constructs the orthogonal projection matrix of the strong interference signal subspace as the interference blocking matrix, uses the matrix to pretransform the global subspace, and then eigen decomposes again to obtain the target DOA. Compared with the traditional algorithm, the algorithm proposed in this paper has a smaller RMSE, and there is no array aperture loss. It functions in a complex and volatile electromagnetic environment.

\section{Data Availability}

The data that support the findings of this study are available from the corresponding author upon reasonable request.

\section{Conflicts of Interest}

The authors declare that there are no conflicts of interest regarding the publication of this study. 


\section{Acknowledgments}

This work was supported by the Natural Science Foundation of China (Grant no. 62071476).

\section{References}

[1] Z. Wang, R. Schaeger, M. Skoglund, M. Xiao, and H. Poor, "Strong secrecy for interference channels based on channel resolvability," IEEE Transactions on Information Theory, vol. 64, p. 1, 2018.

[2] Q. Liu, Y. Gu, and H. C. So, "DOA estimation in impulsive noise via low-rank matrix approximation and weakly convex optimization," IEEE Transactions on Aerospace and Electronic Systems, vol. 55, no. 6, pp. 3603-3616, 2019.

[3] W. Fu, Z. Hu, and D. Li, "A sorting algorithm for multiple frequency-hopping signals in complex electromagnetic environments," Circuits, Systems, and Signal Processing, vol. 39, no. 4, pp. 1-23, 2020.

[4] S. Salari, F. Chan, T. Chan, and R. Guay, "DOA estimation using compressive sampling-based sensors in the presence of interference," IEEE Transactions on Aerospace and Electronic Systems, vol. 99, p. 1, 2020.

[5] Q. Liu, J. Xu, Z. Ding, and H. C. So, “Target localization with jammer removal using frequency diverse array," IEEE Transactions on Vehicular Technology, vol. 69, no. 10, pp. 11685-11696, 2020.

[6] X. Wang, M. Amin, F. Ahmad, and E. Aboutanios, "Interference DOA estimation and suppression for GNSS receivers using fully augmentable arrays," IET Radar, Sonar \& Navigation, vol. 11, no. 3, pp. 474-480, 2017.

[7] H. R. Park and J. Li, "Efficient sparse parameter estimation based methods for two-dimensional DOA estimation of coherent signals," IET Signal Processing, vol. 14, no. 7, 2020.

[8] F. Izedi, M. Karimi, and M. Derakhtian, "Joint DOA estimation and source number detection for arrays with arbitrary geometry," Signal Processing, vol. 140, pp. 149-160, 2017.

[9] Q. Fang, M. Jin, W. Liu, and Y. Han, "DOA estimation for sources with large power differences," International Journal of Antennas and Propagation, vol. 2021, Article ID 8862789, 12 pages, 2021.

[10] Y. Yang, Y. Zhang, and L. Yang, "Wideband sparse spatial spectrum estimation using matrix filter with nulling in a strong interference environment," Journal of the Acoustical Society of America, vol. 143, no. 6, pp. 3891-3898, 2018.

[11] J. Zhang, G. Liao, and J. Zhang, "Weak signal DOA estimation method based on noise subspace expansion under strong signal background," Systems Engineering and Electronics, vol. 31, no. 06, pp. 1279-1283, 2009.

[12] H. Dong, T. Xu, and C. Wang, "Modified projection blocking method for two-dimensional weak signal DOA estimation under strong interference background," Signal Processing, vol. 29 , no. 02 , pp. 221-227, 2013.

[13] D. Xu, M. Li, W. Wang, and X. Wang, "Joint DOD and DOA angle estimation of coherent targets for bistatic MIMO radar," in Proceedings of the Third International Conference on Instrumentation, Measurement, Computer, Communication and Control, pp. 1025-1028, Washington, DC, USA, September, 2013.

[14] H. Li, W. Cui, B. Ba, H. Xu, and Y. Zhang, "Central DOA estimation method for exponential-type coherent distributed source based on fourth-order cumulant," International Journal of Antennas and Propagation, vol. 2020, no. 2, 11 pages, Article ID 8968136, 2020.
[15] M. Tufail and A. Ahmed, "Genetic algorithm-based improved DOA estimation using fourth-order cumulants," International Journal of Electronics Theoretical \& Experimental, vol. 104, 2017.

[16] M. Pan, G. Zhang, Z. Hu, and Q. Zheng, "Real-valued off-grid DOA estimation based on fourth-order cumulants using sparse Bayesian learning in spatial coloured noise," IET Communications, vol. 13, no. 19, pp. 3344-3349, 2019.

[17] H. Shi, N. Ma, and Z. Guan, "A fourth-order cumulants orthonormal propagator rooting method based on toeplitz approximation," EURASIP Journal on Wireless Communications and Networking, vol. 13, no. 4, pp. 409-414, 2020.

[18] Q. Liu, H. C. So, and Y. Gu, "Off-grid DOA estimation with nonconvex regularization via joint sparse representation," Signal Processing, vol. 140, pp. 171-176, 2017.

[19] G. Zheng, Y. Song, and C. Chen, "Height measurement with meter wave polarimetric MIMO radar: signal model and MUSIC-like algorithm," Signal processing, vol. 190, no. 6, pp. 1872-7557, 2021.

[20] L. Qiu, T. Lan, and Y. Wang, "A sparse perspective for direction-of-arrival estimation under strong near-field interference environment," Sensors, vol. 20, no. 1, p. 163, 2019.

[21] Y. Gao, X. U. Jia, and T. Long, "Performance analysis of antijamming method via block matrix," Journal of Signal Processing, vol. 31, 2015.

[22] J. Gong, S. Lou, and Y. Guo, "DOA estimation method of weak sources for an array antenna under strong interference conditions," International Journal of Electronics, vol. 105, no. 9, pp. 1522-1531, 2018.

[23] H. Chen and H. Su, "A new method of DOA estimation in the background of strong interference signal," Chinese Journal of Electronics, no. 03, pp. 530-534, 2006.

[24] H. Chen, C. Hou, Q. Wang, and L. Huang, "Cumulants-based toeplitz matrices reconstruction method for 2-D coherent DOA estimation," IEEE Sensors Journal, vol. 14, no. 8, pp. 2823-2832, 2014.

[25] Y. Wang, M. Trinkle, and B. W. H. Ng, "Two-stage DOA estimation of independent and coherent signals in spatially coloured noise," Signal Processing, vol. 128, no. Nov, pp. 350-359, 2016.

[26] H. Yang, L. Yi, and W. Xi, "DOA estimation of coherent signals on coprime arrays exploiting fourth-order cumulants," Sensors, vol. 17, no. 4, 2016.

[27] Y. Wang, L. Wang, and X. Yang, "Efficient cumulant-based methods for joint angle and frequency estimation using spatial-temporal smoothing," Electronics, vol. 8, no. 1, 2019. 